\title{
Crowdsourcing Physiological Conditions and Subjective Emotions by Coupling Technical and Human Mobile Sensors
}

\author{
Bernd Resch ${ }^{1,2,3}$, Martin Sudmanns ${ }^{1}$, Günther Sagl ${ }^{1,2,4}$, Anja Summa ${ }^{2}$, Peter Zeile ${ }^{5}$ \\ and Jan-Philipp Exner ${ }^{5}$ \\ ${ }^{1}$ Department of Geoinformatics, University of Salzburg, Austria \\ ${ }^{2}$ Institute of Geography - GIScience, Heidelberg University, Germany \\ ${ }^{3}$ Center for Geographic Analysis, Harvard University, USA \\ ${ }^{4}$ Dept of Geoinformation and Environmental Technologies, Carinthia University of Applied Sciences \\ ${ }^{5}$ Computergestützte Planungs- und Entwurfsmethoden (CPE), University of Kaiserslautern, Germany
}

Full paper double blind review

\begin{abstract}
In contrast to previous approaches, Urban Emotions defines Smart Cities not only as technology-enriched cities, but also emphasises the human factor - Smart Citizens. This is due to the fact that the question of how people perceive a city and how they feel about it has always been important in urban planning and management. In this paper we present our approach for crowdsourcing physiological conditions and subjective emotions by combining data from technical sensors (measuring psycho-physiological parameters) and human sensors ("People as Sensors" contributing subjectively perceived emotions). Furthermore, we couple this crowdsourcing approach with a technical architecture to harmonise and integrate the sensor data via standardised service interfaces (Sensor Web Enablement - SWE) to allow for generic access for further analysis and visualisation. Finally, we discuss the use of emotion information in urban planning and point out related privacy issues together with a number of strategies to mitigate those.
\end{abstract}

\section{Introduction}

Smart Cities have been widely defined as technology-enriched cities that become more intelligent through the use of ICT (CHOURABI et al. 2012). Yet, the human factor (Smart Citizens), which is indispensable for ultimately making a city smart, has been neglected, even though the question of how people perceive a city and how they feel about it has always been an important issue in urban planning and management. This is underpinned by Jane Jacobs' quote that "cities have the capability of providing something for everybody, only because, and only when, they are created by everybody" (JACOBS 1961).

Jacobs' quote supports the need of a participatory spatial planning approach for a "good" and citizen-centric planning. Thus, it is important to further investigate how citizens perceive urban space, how they react to it and what ways exist to extract their feelings and opinions. This includes theoretical visions as well as human-centred implementation strategies involving emotion data (ZEILE et al. 2015). Thus, emotion information can poten- 
tially enrich a variety of areas related to urban planning, including traffic planning, urban safety, people-centric tourism, or assessing previous planning measures (KAISER et al. 1995).

Traditionally, emotion data are collected in interviews and surveys. However, those methods are complicated, error-prone, and unreliable (PITTA et al. 2006). On the contrary, the integration of technical and human emotion sensors complemented by direct feedback from citizens ("People as Sensors" or "eDiaries") to stakeholders via real-time participatory communication channels, such as Citizen Sensing, has enabled new insights into urban patterns and dynamic contexts like mobility, Quality of Life (QoL), or public safety.

Against this background, Urban Emotions proposes a human-centred approach interfacing the research fields of geoinformatics, computer science, computational linguistics, sensor technology, citizen science, and spatial planning. More precisely, Urban Emotions focuses on a new and human-centric perspective of the city, in which humans as the "users of a city" represent the principal sensing element. Therefore, we combine and merge objective elements of sensor technology with subjective measurement methods to create a "human sensor network". The underlying motivation is to generate a new information layer for planners, visualising the measured spatial perception. These visualisations allow conclusions about human behaviour in urban environments, and enable a new citizen-centred perspective in planning processes.

In this paper we present our approach for crowdsourcing physiological conditions (technical sensors measuring psycho-physiological parameters) and subjective emotions (human sensors "People as Sensors" contributing subjectively perceived emotions). Further, we propose a technical architecture, as these data need to be harmonised and integrated into a standardised Spatial Data Infrastructure (SDI) to allow generic access for further analysis and visualisation in urban planning processes.

The structure of the paper is as follows. Section 2 presents a number of related approaches in the areas of mobile applications for collecting physiological data and the use of emotion information in urban planning. Thereafter, we present our approach from a theoretical viewpoint, i.e., the requirements for assessing emotions using technical and human sensors, interaction design for a related People as Sensors app, and standardised integration of measurements into an SDI. Section 4 lays out the prototypical realisation, before section 5 presents its use in urban planning, including privacy issues to be considered. Finally, we end our paper with a set of key conclusions.

\section{Related Work}

The proposed approach is placed at the intersection of technical and planning domains. Therefore, related work from both areas - mobile applications for collecting physiological data and the use of physiological measurements in urban planning - has to be discussed.

\subsection{Mobile Apps for Collecting Physiological Data}

Mobile applications, which measure physiological conditions, are currently widely used in the field of health care. Estimates for the strongly growing number of apps range from 
1,500 to more than 7,000 (TERRY 2010), depending on the definition of a "health-related application". A subset of these apps collects data that are measured by an additional (wearable) device like a biosensor. Collecting health data and body conditions with mobile devices is widely established (PITTA et al. 2006). Yet, collecting subjective feelings of people is usually done using conventional diaries and questionnaires, even though it is well known that questionnaires are usually applied long after the event occurred and that they are difficult to create and to analyse. Due to their design and application, questionnaires ask the test person to recall events which occurred minimum one hour ago (PITTA et al. 2006). Therefore, instant on-site collection using questionnaires is virtually impossible and the exact location information of an event may get lost or is difficult to resemble.

In contrast to previous mobile apps in health care that usually aim to help and support one individual, Urban Emotions seeks to collect crowdsourced data for further analysis. Furthermore, the location of the person is vital information in the context of Urban Emotions, whereas it has not played an important role in most previous use cases. Urban Emotions considerably extends the state of the art in this regard by analysing personal data in an anonymous way (aggregated data instead of individual datasets - s. sub-section 5.2) to complement current urban planning purposes.

\subsection{Using Physiological Data in Urban Planning}

The question of how people perceive their environment has been a special field of research since the late 1950s. It has been outlined that human perceptions and the urban space are closely linked as each situation creates its own special atmosphere and triggers an emotional reaction in the observer (MODY et al. 2009). This led to the "biomapping" concept, a digital map with georeferenced emotion data (NOLD 2009). Even though this research does not permit the conclusion that emotions can be directly transferred into categorical values for urban planning, it was a first step to fertilize the scientific debate about mapping emotions. Additionally, within the last decades, new developments enabled the combination of traditional urban planning with new technologies and methods (sensor networks, crowdsourcing, etc.). Moreover, the use of mobile devices with special apps, which are nowadays omnipresent in citizens' lives, allows for expressing feelings at virtually any location at any time. One example is the app "Mapiness", whose aim is to understand how people's feelings are affected by features of their current environment, including air pollution, noise and green spaces (MACKERRON \& MOURATO 2012).

As a result of these considerations, the question arises how planners can recognise citizens' problems in a spatial context, i.e., a new kind of participation is required. Here, Urban Emotions provides new information layers for planning processes. This can only be achieved by noting the works of (CASTELLS 1996) concerning a network society, pointing out a fundamental change in the understanding of planning including, for instance, bottomup processes of participation that proactively involve citizens in urban acting. The extension of the repertoire of methods in spatial and urban planning with "sensor technologies" is key, as traditional deductive planning has to be complemented with inductive planning approaches, such as crowdsourcing emotion data. 


\section{Concept: Crowdsourcing Physiological and Subjective Emotions}

Taking the conceptual considerations, which will be explained in sub-sections 3.1-3.3, into account, a basic architecture for crowdsourcing emotions and integrating them into a SOAbased Spatial Data Infrastructure (SDI) can be divided into two modules, as shown in Figure 1; first, the collection of emotion data (measured psycho-physiological data and subjective emotions), and second, the integration of this information via standardised interfaces (OGC SWE) (BRÖRING et al. 2011) to store data in an SDI for further analysis and visualisation.

From a technical viewpoint, the architecture comprises the communication between three components: psycho-physiological sensors, a mobile device like a smartphone, and a server including the measurement database. The mobile application itself provides connections to different sensors via Bluetooth LE (low energy) and the standardised transmission of the harmonised measurements to a database, which occurs in in predefined intervals.

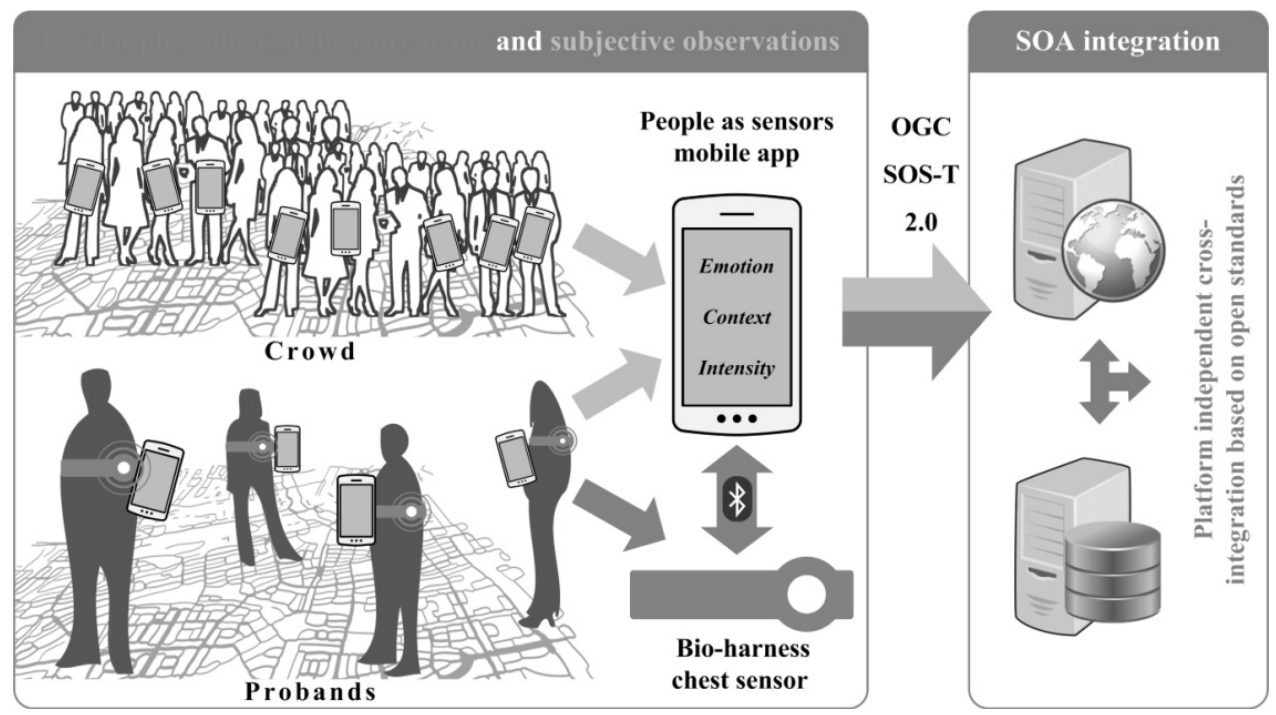

Fig. 1: Crowdsourcing Emotion Information through Technical and Human Sensors

\subsection{Assessing Emotions Using Technical and Human Sensors}

As laid out above, the Urban Emotions concept assesses psycho-physiological data (acquired by technical sensors) and subjective sensations (contributed by probands). The psycho-physiological measurements cover a variety of parameters including pulse, heart rate, heart rate variability, additional heart rate, skin temperature, and others. These measurements are then correlated with the subjective feelings specified by the test persons.

Measuring emotions using psycho-physiological sensors is still challenging from a psychological and a physiological viewpoint. In the last years though, a number sensors became 
capable of reliably measuring human body conditions. Some of them have been widely used and successfully tested by athletes, but also for people in complex and stressful situations like emergency responders, soldiers, or astronauts. A sensor, which has been proven to be reliable in operation and regarding the quality of the measurements, is the Zephyr BioHarness 3 (HAILSTONE \& KILDING 2011), which we also use in our approach.

The use of the sensors for Urban Emotions differs from the usual application areas for which they have been designed (monitoring human performance), in that additional processing requirements have to be considered and managed. In order to make use of the measured data, they need to be harmonised with the user's current location, which is provided by the mobile device, and the current time. Furthermore, the data have to be stored in a central database for further analysis and visualisation - and not only stored locally. Finally, the data need to be transmitted in near real time in an aggregated way to prevent data loss and to eliminate privacy issues.

\subsection{Interaction Design of the People as Sensors Smartphone App}

As mentioned above, the purpose of the People as Sensors app is twofold: 1.) to collect physiological sensor data; and 2.) to collect subjective sensations. The app is designed modularly, which on the one hand allows usage of the subjective input functionality also without technical sensors being coupled, and on the other hand it provides a simple mechanism for new sensors to be coupled (s. sub-section 3.3).

To be usable for further geo-analysis, measurements need to be preprocessed, harmonised, and continuously checked. This allows the user to be instantly prompted for their input of a subjectively perceived emotion after the sensor has detected the exceedance of a predefined threshold. This notification has to be shown immediately (temporally and spatially close to the event) to be able to link the user input with the physiological measurement. This is important because it is not always possible to derive complete emotion data purely from sensor measurements without additional information provided by the user, which in turn is essential to understanding the underlying process that caused an emotional spike. This additional information comprises the person's actual emotion (the type of emotion), the cause why an emotion occurred (the context of the emotion), and the emotion's intensity.

In the context of Urban Emotions, the user is prompted to insert subjective emotion information in three different circumstances - apart from the fact that the user can contribute their current sensations at any time; 1.) a trigger notifies the user when the application detects significant values of the physiological measurements; 2.) a trigger notifies the user when the user crosses a geo-fence, i.e., a predefined area against which the application checks its current position; and 3.) a time dependent trigger notifies the user in predefined intervals.

\subsection{Standardised Measurement Integration: Transactional Sensor Web Enablement}

The architecture of the app needs to allow the use of different sensors by ensuring integrability of a variety of sensor protocols. In other words, coupling of different sensor technologies and protocols needs to be easily feasible by using a pluggable module for each sensor. Each of these modules translates the sensor-specific formats into a harmonised data 
structure, the standardised Observations and Measurements (O\&M) format, and sends it as an InsertObservation request, which is specified in the Sensor Observation Service (SOS-T 2.0) of the OGC (BRÖRING et al. 2011).

This concept is in line with the goal of SWE to access and manage different kinds of sensors via the Internet in a standardised way. This allows clients to discover, manage and receive data from sensors without a priori knowledge of the sensor systems (BRÖRING et al. 2011). Once the data are harmonised and accessible using a service-oriented architecture (SOA), the different sensor technologies and their individual data structures can be accessed using standardised protocols.

\section{$4 \quad$ Validation People as Sensors: Prototypical Proof of Concept}

In the design of a mobile app for collecting subjective emotions, several design considerations (sub-section 4.1) and aspects of user interaction (sub-section 4.2) were taken into account. During the design process, these deliberations have been coordinated with environmental psychologists and urban sociologists.

\subsection{Design Considerations}

In the design of the app, we aimed to fulfil common guidelines for mobile applications. First, we applied the design principles: "make it direct", "keep it lightweight", "stay on the page", "provide an invitation", "use transitions", and "react immediately", as defined by (SCOTT \& NEIL 2009). To satisfy these principles, we designed a simple interface that offers users the possibility to first input the type and the context of the emotion. In order to comply with the requirement of immediate feedback to the user, we show a map containing the emotions of a city right after a user has entered their information (RESCH et al. 2015). Like this, the motivation for entering emotion information is kept high and users can immediately "compare" their own impressions with other persons' perceptions.

The interface is lightweight with respect to design elements, the number of "clicks" required, and the information provided to the user (RESCH et al. 2015). To comply with the requirement of displaying an invitation, we notify the user when their input is required (s. sub-section 3.2). More, we greatly avoid strong colouring of the app and assigning colours to emotions in order to preclude emotional biases (MOHAMMAD 2011).

\subsection{User Interface and User Interaction}

Figure 2 shows the four screens with the lists for emotion, the context, the intensity slider, and the immediate feedback. 


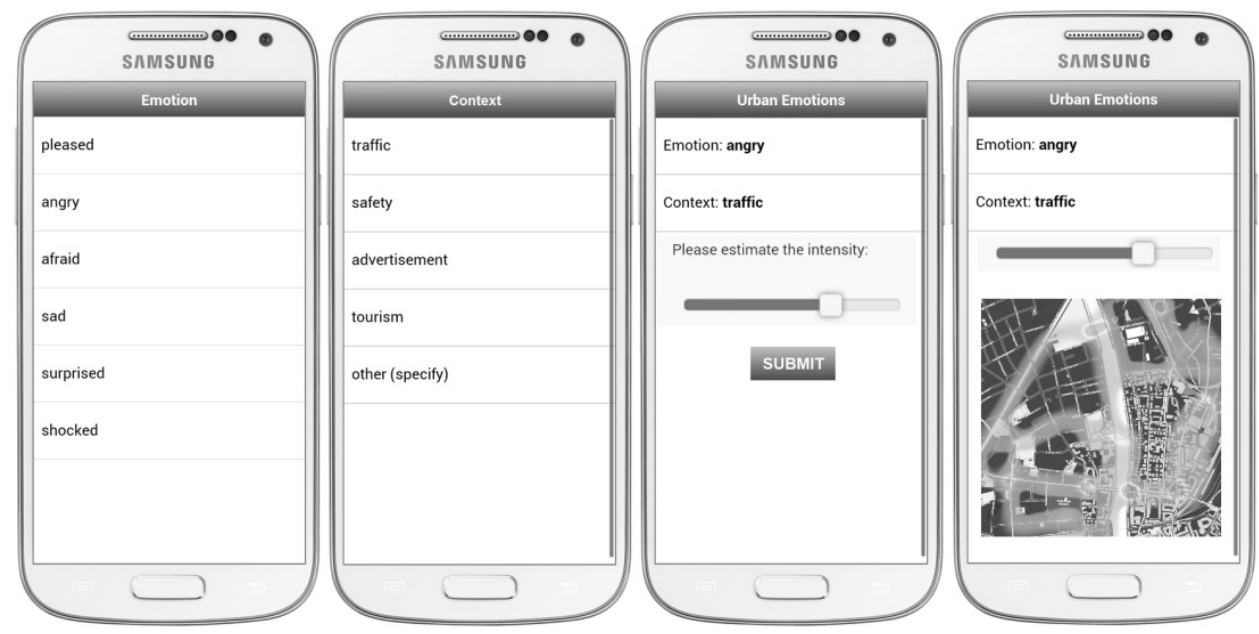

Fig. 2: User Interface of the "People as Sensors" Smartphone App

The guidance through the user interaction dialogue consists of five steps:

\section{1.) Notification}

As mentioned above, notifications are used to ask the user to enter information about their emotion at their current location. The point in time when a notification is sent can be based on three different triggers: threshold exceedance of physiological measurements, the user crossing a geo-fence, or notifications in a predefined interval. The user can choose between vibration, audio or visual alerting, or a combination of these. In this way, the user is free to leverage the type of notification that is most advantageous for their current context (the physical context, surrounding noise levels, how and where the smartphone is carried, etc.).

\section{2.) Emotion, context and intensity}

Second, the user is asked to insert three types of information, which are emotion type, context, and intensity. For the emotion type, the user can choose from a set of predefined values: pleased, angry, afraid, sad, surprised, and shocked (different emotion models exist). Next, the user is forwarded to the context screen, which asks for the reason why an emotion occurred. Again, the user can choose from a list of predefined values, which are adaptable depending on the concrete use case. In addition, the app offers the insertion of a new context if the provided list does not contain an appropriate one. Finally, the intensity of the emotion can be entered using a slider bar in a value range between 1 and 100 .

\section{3.) Geolocation \& time}

Since it might not be possible for the user to instantly react to the notification and insert the data instantly after the event, the app needs to estimate the geolocation and the time at which the event occurred. Thus, for reasons of data robustness, one position and three timestamps are associated with a measurement. First, the internally computed geolocation and timestamp of the mobile device when the event took place are sent. However, the location information of the mobile device might be outdated due to problems in the measurement (e.g., when a person is indoors and the GPS measurements are only resumed 
when the user returns outdoors). Therefore, the second timestamp is the time when the position was measured to store the information how old the location information is. The third timestamp is when the user eventually has inserted all values into the People as Sensors app and submits the data to the server. Through the combination of these timestamps, the application estimates how confident the user was in giving their subjective input by analysing the time difference between the sensor measurement and the user input.

\section{4.) Transmission to the server}

Once the data are processed, they are transformed into the OGC O\&M data format and sent to an SOS via an InsertObservation request, as sketched out in sub-section 3.3.

\section{5.) Result visualisation}

Compared to other apps, the Urban Emotions app stays rather unimpressive to the user to avoid psychological distractions and bias (s. sub-section 4.1). To keep the user's interest and willingness to participate high, the physiological data are visualised in near real time directly on the mobile device to provide a direct feedback and incentive. Concretely, the user immediately receives visual feedback in the form of a map showing their position together with their inputs as a comparison with other users at the same location.

\section{Use in Urban Planning Processes and Privacy Issues}

The presented approach is one essential part in the Urban Emotions concept, directly at the interface between geoinformatics and urban planning. In the following sub-sections, we lay out its practical significance in urban planning and privacy issues related to personal data.

\subsection{Emotion Information Enriching Urban Planning}

Urban Emotions is a new anthropocentric approach to analyse and survey interactions between the dynamics in time, space, and humans' perceptions and emotions. Especially urban design processes, but also safety issues in traffic planning are a new application field for these surveys. Results are part of recommendations during weighting in decision processes if stakeholders are open-minded for new innovative methods of a citizen-centric urban planning. Furthermore, the creation of contextual emotion information can be used in monitoring processes for a validation of sustainable planning projects. For example, people's emotion levels can be measured before and after a traffic construction project.

Nevertheless, Urban Emotions is not thought as a general tool of solving all planning tasks, but it can help create another view and better understanding of the "urban corpus" (ZEILE et al. 2015). As mentioned before, it will be a success if this new layer of knowledge will be integrated as an indicator system in official planning processes. It can deliver valuable information not for everything and in general, but for special tasks like the discussion of subjectively perceived safety or in design processes.

\subsection{Privacy Issues with Personal Data}

The use of crowdsourced personal data poses several challenges to protect the users' privacy. One challenge is the implementation of privacy-preserving technologies in order to 
protect personal data against unintended transfer or against misuse by a third party (BARCENA et al. 2014). Since personal geo-data are highly individual, the user can be reidentified, even if the data is anonymised, for instance by analysing the working place and the living place locations, which are a unique for most of the people. DE MONTOYE et al. (2013) show that only four random positions of a track are needed to identify individuals.

In this context, a central concept is "location privacy" which describes the ability of an individual to move in public space when their position is not collected and stored. The most restrictive way to achieve location privacy and to avoid misuse of personal data is opting out and thus prohibiting the collection of data at all (BLUMBERG \& ECKERSLEY 2009), which in many cases is not an option though. Due to the numerous possibilities to analyse geographic data and extract information, it is assumed that one third of the free apps collect location information (KERSTEN \& KLETT 2012). For applications that need to collect and store personal information, the Electronic Frontier Foundation and others recommend the anonymisation of the data, and the usage of strong cryptographic protocols.

Yet nowadays, $20 \%$ of all mobile apps are sending data from a mobile sensor to a smartphone in an unencrypted fashion (e.g., via Bluetooth) and are therefore seen as vulnerable for unintended data leakage (BARCENA et al. 2014). When the source code of an app is not openly available or it uses third-party-code, it is difficult for the user to gain control of which resources are accessed, how the data are processed, and which data are sent to which server. This can be a serious issue, since $47 \%$ of the free Android apps are using thirdparty-code (KERSTEN \& KLETT 2012).

The willingness to voluntarily share personal data is linked with the trust in the security of the data. In contrast, it seems that many users tend to share private information and do not actively try to protect it, even if they claim that they are concerned about their privacy (ACQUISTI et al. 2013). An example is the high number of users of social networks, even though users do not have much influence on data collection and processing policies. This is particularly due to the operators' claims that less privacy is a new social norm and is worth less than better service for the user.

Another question that arises in the particular setting of Urban Emotions is how test persons' answers are affected by the smartphone's notification, as they need to shift their focus to the app for inputting their subjectively perceived emotions after they had been notified. From a psychological viewpoint, this may induce two kinds of biases: first, conditioning effects (people might input what they think is expected in a recurring situation), and second, dilutions due to cognitive processes rather than a spontaneous answer (as a result from a delay between the sensor measurement and the input in the People as Sensors app). Furthermore, this way of collecting subjective emotions might result in dangerous situations depending on the use case (e.g., people need to stop abruptly when riding a bike).

To tackle the above-mentioned privacy issues, the Urban Emotions app has inherently built in a number of Privacy by Design aspects. First of all, it does not collect data which are directly linked to an individual user, as users do not need to enter their name or register in order to use the app. Second, the data, which are collected by a user, are linked to a unique identifier which is not based on personal data. Therefore, the data are stored in an anonymised way. Third, we further anonymise data by splitting trajectories in non-reconstructable sub-paths to prohibit identity derivation. Fourth, no proprietary and closed-source third-party code is used in the app itself (sensor software is mostly closed source though). 
Fifth, data are only analysed in an aggregated fashion as the goal of the approach is to detect hot spots throughout the whole city rather than analysing individual user behaviour. Finally, all test persons participate voluntarily in our studies through an opt-in agreement undergoing a thorough instruction phase, knowing that their data will be collected and analysed.

\section{Conclusion and Outlook}

In contrast to previous approaches, Urban Emotions defines Smart Cities not only as technology-enriched cities, but emphasises the human factor - "Smart Citizens". This tries to answer the pertaining question of how people perceive a city and how they feel about it, which has always been an important issue in urban planning and management.

In this paper we presented our approach for crowdsourcing physiological conditions and subjective emotions by combining human emotions derived from technical sensors (by measuring physiological parameters) and subjectively perceived emotions (through a "People as Sensors" smartphone app). Furthermore, we coupled this crowdsourcing approach with a technical architecture to harmonise and integrate the sensor data via standardised service interfaces (Sensor Web Enablement - SWE) to allow for generic access for further analysis and visualisation in urban planning processes. Using the Urban Emotions app, citizens can enter their subjective sensations concerning a particular place in the city. This input is then combined with calibrated sensor measurements for crossvalidation. Apart from user-initiated inputs, three different types of trigger dedicatedly ask the user for their subjective sensations: threshold-based, location-based, or based on regular time intervals.

In the next design phase, we aim to improve the app's input modalities. One option that we are currently investigating is the use of a "colour wheel" (comparable to the RGB colour wheel) to enable the input of a combined emotion in a quasi-continuous emotion space instead of just a single emotion. Even though this does not comply with the idea of avoiding bias (s. above), it could be important to allow for inputting several emotions, as perceived emotions are often not assignable to a single category. Furthermore, we are currently carrying out distinct use cases to further explore and validate practical use in urban planning - next to the prototypical experiments presented in (RESCH et al. 2015). In addition, we are currently intensifying our collaboration with emotion psychologists to optimise the assessment of emotions through the combination of physiological sensors and eDiaries. First results of ongoing studies show that the combination of quantitative measurements and qualitative interpretations of the urban environment can potentially create a new layer of information for planners, enriching the planning participation process.

\section{Acknowledgements}

The authors would like to express their gratitude to the German Research Foundation (DFG - Deutsche Forschungsgemeinschaft) for supporting the project "Urban Emotions", reference number ZE 1018/1-1 and RE 3612/1-1. This research has been supported by the Klaus Tschira Stiftung gGmbH. 


\section{References}

Acquisti, A., John, L. K. \& Loewenstein, G. (2013), What Is Privacy Worth? The Journal of Legal Studies, 42 (2), 249-274.

Barcena, M. B., Wueest, C. \& LAU, H. (2014), How Safe Is Your Quantified Self. Symantech: Mountain View, CA, USA.

Blumberg, A. J. \& Eckersley, P. (2009), On Locational Privacy, and How to Avoid Losing It Forever. Electronic Frontier Foundation.

Bröring, A., Echterhoff, J., Jirka, S., Simonis, I., Everding, T., Stasch, C., Liang, S. \& Lemmens, R. (2011), New Generation Sensor Web Enablement. Sensors, 11 (3), 2652-2699.

Castells, M. (1996), The Rise of the Network Society: Volume I: the Information Age: Economy, Society, and Culture. Blackwell Publishers.

Chourabi, H., Nam, T., Walker, S., Gil-Garcia, J. R., Mellouli, S., Nahon, K., PARdo, T. A. \& Scholl, H. J. (2012), Understanding Smart Cities: An Integrative Framework. In 2012 45th Hawaii International Conference on System Sciences. IEEE, 2289-2297.

Hailstone, J. \& Kilding, A. E. (2011), Reliability and Validity of the Zephyr BioHarness to Measure Respiratory Responses to Exercise. Measurement in Physical Education and Exercise Science, 15 (4), 293-300.

JACOBS, J. (1961), The Death and Life of Great American Cities. Random House LLC, New York, USA.

Kaiser, E., Godschalk, D. \& Chapin, F. (1995), Urban Land Use Planning. 4th Ed. University of Illinois Press, Urbana IL, USA.

Kersten, H. \& Klett, G. (2012), Mobile Device Management. Hüthig Jehle Rehm, Heidelberg/München/Landsberg/Frechen/Hamburg.

Mody, R. N., Willis, K. S. \& Kerstein, R. (2009), WiMo: Location-based Emotion Tagging. In: Scenario. ACM, 1-4.

Mohammad, S. (2011), Even the Abstract Have Colour: Consensus inWord-Colour Associations. In: Proceedings of the 49th Annual Meeting of the Association for Computational Linguistics, 368-373.

De Montjoye, Y.-A., Hidalgo, C. A., Verleysen, M. \& Blondel, V. D. (2013), Unique in the Crowd: The Privacy Bounds of Human Mobility. Scientific Reports, 3.

NolD, C. (2009), Emotional Cartography: Technologies of the Self. Christian Nold.

Pitta, F., Troosters, T., Probst, V. S., Spruit, M. A., Decramer, M. \& Gosselink, R. (2006), Quantifying Physical Activity in Daily Life with Questionnaires and Motion Sensors in COPD. European Respiratory Journal, 27 (5), 1040-1055.

Resch, B., Summa, A., SAgl, G., ZeIle, P. \& ExNER, J.-P. (2015), Urban Emotions - GeoSemantic Emotion Extraction from Technical Sensors, Human Sensors and Crowdsourced Data. In: GARTner, G. \& HuAng, H. (Eds.), Progress in Location-Based Services 2014. Springer International Publishing, 199-212.

Scott, B. \& NeIL, T. (2009), Designing Web Interfaces: Principles and Patterns for Rich Interactions. O'Reilly Media, Sebastopol, USA.

TERry, M. (2010), Medical Apps for Smartphones. Telemed JE Health, 16 (1), 17-22.

ZeILe, P., Resch, B., EXNER, J.-P. \& SAGL, G. (2015), Urban Emotions - Benefits and Risks in Using Human Sensory Assessment for Extraction of Contextual Emotion Information. In: Planning Support Systems and Smart Cities. Springer, Heidelberg, Germany, pending. 